\title{
Cholinergic Neurons in Nucleus Subputaminalis in Primary Progressive Aphasia
}

\author{
Hayam Hamodat, John D. Fisk, Sultan Darvesh
}

\begin{abstract}
Background: Primary Progressive Aphasia (PPA) is a syndrome characterized by an isolated impairment of language function at disease onset. The cholinergic system is implicated in language function and cholinergic deficits are seen in the brains of individuals with PPA. One major source of cholinergic innervation of the cerebral cortex is the nucleus basalis of Meynert (NBM) within which lies the nucleus subputaminalis (NSP). This nucleus is postulated to be involved in language function. We compared the abundance of cholinergic neurons in the NBM and NSP of controls and individuals with PPA. Also explored was whether the individuals presenting with PPA, who subsequently developed different clinical and neuropathological profiles, showed similar cholinergic deficits in the NSP. Methods: Cytoarchitecture of the basal forebrain was studied using Nissl staining in control $(n=5)$ and PPA $(n=5)$ brains. Choline acetyltransferase (ChAT) immunohistochemical staining labeled cholinergic neurons were quantified using Neurolucida software. Results: In comparison to matched controls, PPA showed reduction of cholinergic neurons in the NBM $(t(8)=4.04, p=0.0037$; Cohen's effect size value $d=2.62)$ and the NSP $(t(6)=4.62, p=0.0042$; Cohen's d effect size $d=2.92)$. The average percent of cholinergic neuronal loss was relatively higher in the NSP (64.7\%) compared to the NBM (47.7\%). Conclusion: Regardless of underlying pathology, all cases presenting with PPA showed a marked loss of cholinergic neurons in the NSP, providing further evidence for the importance of this nucleus in language function.
\end{abstract}

RÉSUMÉ : Pertes de neurones cholinergiques dans le nucleus subputaminalis d'individus atteints d'aphasie primaire progressive. Contexte : L'aphasie primaire progressive (APP) constitue un syndrome qui se caractérise par un trouble isolé de la fonction langagière au moment où la maladie manifeste ses premiers signes. On le sait, le système cholinergique est lié à la fonction langagière. Voilà pourquoi des déficits cholinergiques sont observés dans les cerveaux d'individus atteints d'APP. Une source majeure d'innervation cholinergique du cortex cérébral se situe dans le noyau basal de Meynert (NBM), noyau dans lequel on trouve le nucleus subputaminalis (NSP). On tend à penser que ce dernier est impliqué dans la fonction langagière. Nous avons ainsi comparé l'abondance de neurones cholinergiques dans le NBM et le NSP chez des patients atteints d'APP et des témoins. Nous avons aussi tenté de savoir dans quelle mesure les individus atteints d'APP ayant subséquemment développé divers profils cliniques et neuro-pathologiques donnaient à voir les mêmes déficits cholinergiques au niveau du NSP. Méthodes : La cytoarchitectonie du prosencéphale basal a été étudiée au moyen de la méthode de coloration de Nissl. Pour ce faire, 5 cerveaux de témoins et 5 cerveaux d'individus atteint d'APP ont été sélectionnés. De la coloration immunohistochimique à base de choline acétyltransférase (ChAT) a permis d'identifier les neurones cholinergiques. Pour les quantifier, nous avons utilisé le logiciel Neurolucida. Résultats : En comparaison avec les témoins, les individus atteints d'APP ont montré une réduction de leurs neurones cholinergiques dans le NBM $(t(8)=4,04 ; p=0,0037$; taille d'effet proposée par Cohen et notée $d=2,62)$ et le NSP $(t(6)=4,62 ; p=0,0042 ;$ taille d'effet proposée par Cohen et notée $d=2,92)$. Le pourcentage moyen de perte de neurones cholinergiques s'est révélé relativement plus élevé dans le cas du NSP (64,7\%) en comparaison avec le NBM (47,7\%). Conclusion : Sans égard à la présence d'une pathologie sous-jacente, tous les individus atteints d'APP ont montré une perte marquée de neurones cholinergiques dans leur NSP, ce qui apporte une preuve supplémentaire quant à l'importance de ce noyau eu égard à la fonction langagière.

Keywords: Basal forebrain, Nucleus of Ayala, Alzheimer's disease, Progressive supranuclear palsy, Vascular dementia, Pick's disease, Choline acetyltransferase

doi:10.1017/cjn.2019.6

Can J Neurol Sci. 2019; 46: 174-183

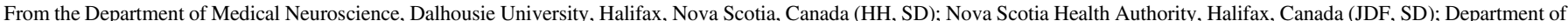

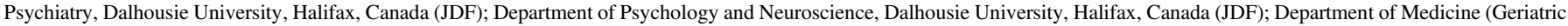

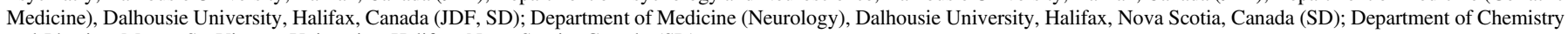
and Physics, Mount St. Vincent University, Halifax, Nova Scotia, Canada (SD).

Received October 29, 2018. Final Revisions Submitted December 19, 2018. Date of Acceptance January 11, 2019.

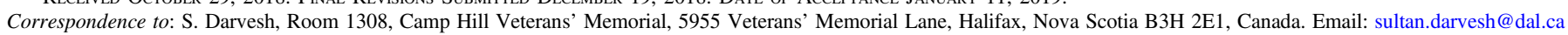




\section{INTRODUCTION}

Several pathologically distinct neurodegenerative disorders display language disturbances. Language deficits may be the presenting symptom in individuals with Alzheimer's disease (AD) ${ }^{1}$ Pick's disease (PiD), ${ }^{2}$ Progressive Supranuclear Palsy (PSP) and corticobasal degeneration (CBD). ${ }^{3}$ Primary Progressive Aphasia (PPA) is a clinical syndrome characterized by isolated impairment in language function, with the relative sparing of other cognitive domains at disease onset. ${ }^{4-7}$ Structural and metabolic imaging studies of PPA have consistently shown asymmetric focal atrophy and hypometabolism in the language dominant (usually left) brain hemisphere. ${ }^{8-12}$

Three predominant patterns of language dysfunction have been described as occurring in individuals with PPA. Those with the nonfluent/agrammatic variant of PPA display effortful speech and spared single word and object comprehension, those with the semantic variant of PPA have impaired naming and single-word comprehension, and those with the logopenic variant of PPA have word retrieval and sentence repetition deficits. ${ }^{6}$ Clinicopathologic studies have most often linked nonfluent PPA to tau-positive pathology, semantic PPA to ubiquitin-positive, TDP43-positive pathology, and logopenic PPA to AD pathology. ${ }^{13}$ Irrespective of the underlying neuropathology, deficits in the cholinergic system have been reported in PPA. ${ }^{14}$ Even in young healthy individuals, it has been shown that administration of the anticholinergic agent, Scopolamine, leads to impairments in reading, writing, verbal fluency, and object naming. ${ }^{15}$

One potential source of cholinergic input to cortical regions involved in language function is the nucleus basalis of Meynert (NBM). ${ }^{16,17}$ The NBM is a prominent structure found on the ventral surface of the brain that contains large hyperchromic cells, approximately $90 \%$ of which are cholinergic. ${ }^{18}$ These cholinergic neurons, designated as $\mathrm{Ch} 4$, project to the amygdala and to the entire cortical mantle. ${ }^{18-22}$ The NBM region has been subdivided based on projection patterns and can be identified by neuroanatomical landmarks. ${ }^{17,18,20}$ The cholinergic NBM has been subdivided into several regions designated as Ch4 sectors. ${ }^{18}$ These include: anterolateral (Ch4-al), anteromedial (Ch4-am), intermediodorsal (Ch4-id), interomedioventral (Ch4-iv), anterointermediate (Ch4ai), and posterior (Ch4p). Neurons in the posterior subdivision of $\mathrm{Ch} 4$ and $\mathrm{Ch} 4 \mathrm{p}$ predominantly project to the superior and polar temporal cortical regions and to the language areas. ${ }^{17}$ Based on anatomical landmarks, in coronal sections, the nucleus subputaminalis (NSP), also called as the nucleus of Ayala, ${ }^{23}$ is located ventral to the putamen from the level of the decussation of the anterior commissure to the level of mammillary bodies. The NSP has only been described in humans and anthropoid monkeys. ${ }^{24}$ Its proximity to the external capsule suggests connectivity to cortical language areas, and a role in language function in the human brain. ${ }^{16,17,25}$ Previous volumetric analysis of MRI scans of PPA patients has found predominant atrophy of the left hemisphere basal forebrain, including the NSP region, irrespective of the underlying neuropathology. ${ }^{25,26}$

The goals of this study were to examine cholinergic neurons in the NSP of individuals with PPA, and to examine whether individuals whose initial clinical presentation was PPA, but who subsequently had different clinical and neuropathological diagnoses, show similar involvement of cholinergic neurons in the NSP. Using choline acetyltransferase (ChAT) immunohistochemistry, we compared the abundance of cholinergic neurons, in the NSP, between those presenting with PPA and controls, to determine if PPA is associated with a loss of cholinergic neurons in the NSP.

\section{Materials and Methods}

\section{Brain Tissues}

A total of 10 post-mortem human brain tissues ( 5 presenting clinically as PPA and 5 controls), whose demographics are summarized in Table 1, were obtained from The Maritime Brain Tissue Bank (Halifax, Nova Scotia, Canada) following ethical approval from the Nova Scotia Health Authority Research Ethics Board.

\section{Case Descriptions}

\section{Vascular Dementia (VaD)}

A 86-year-old female was presented with language disturbance and was diagnosed with PPA. Her CT/MRI scan showed generalized atrophy and white matter changes. Over a 4-year period, she developed dementia and subsequently died of pneumonia. At autopsy, her brain weighed $1230 \mathrm{~g}$. The left hemisphere was used for neuropathological examination. This showed cerebral cortical microangiopathy, cortical microvascular proliferation, and a recent left cerebellar hemisphere infarct. No regionalization of vascular changes was seen. There was no evidence for Alzheimer pathology, Lewy bodies, or Pick bodies. The right hemisphere was available for examination of the NBM, including the NSP, in this study.

\section{Progressive Supranuclear Palsy (PSP)}

A 93-year-old right-handed male was diagnosed with mild cognitive impairment and word-finding difficulties. Two years later, he had more difficulties in speech with inappropriate words and hesitant fragmentation of sentence structure. Later that year, he was diagnosed with non-fluent PPA. His brain CT scan showed parenchymal atrophy and small vessel ischemic changes. He died of coronary artery disease 4 years after presentation. At autopsy, his brain weighed $1391 \mathrm{~g}$. The right hemisphere was used for neuropathological diagnosis. The neurofibrillary tangles (NFTs) extended into temporal neocortex (Braak stage Ill-VI). The tau pathology was diagnostic for PSP. NIA-AA ABC score ${ }^{27}$ of $\mathrm{B} 1 / \mathrm{CO}$ indicated that $\mathrm{AD}$ neuropathologic change was insufficient to explain dementia in this case. Immunohistochemical staining for $\alpha$-synuclein did not reveal any significant accumulation of Lewy bodies or Lewy neurites. The left hemisphere was available for examination of the NBM, including the NSP, in the present study.

\section{Pick's Disease (PiD)}

A 74-year-old right-handed male developed speech problems and indicated that he had difficulty in thinking and "terrible memory". His wife reported approximately a 1-year history of progressive language difficulty, social withdrawal, no longer initiating conversations or using a telephone, problems with filling out forms, and signing his name. His clinical diagnosis was non-fluent PPA. His brain MRI scan showed generalized cerebral and cerebellar atrophy and non-specific ischemic white matter changes. Four years later, he developed memory problems. 


\section{Table 1: Case demographics}

\begin{tabular}{|c|c|c|c|c|c|c|c|}
\hline Diagnosis & Sex & Age (year) & $\operatorname{PMI}(h)^{a}$ & TIF $^{\mathbf{b}}$ (days) & Brain Weight (g) & Cause of Death & Diagnosis \\
\hline \multicolumn{8}{|c|}{ Primary Progressive Aphasia } \\
\hline $\mathrm{VaD}$ & $\mathrm{F}$ & 86 & 20 & 1.97 & 1230 & Pneumonia & Vascular dementia \\
\hline PSP & $\mathrm{M}$ & 93 & $<24$ & 4.81 & 1391 & Unknown & $\begin{array}{l}\text { Progressive } \\
\quad \text { supranuclear palsy }\end{array}$ \\
\hline $\mathrm{PiD}$ & M & 74 & 10 & 3.02 & 1100 & Heart failure & Pick's disease \\
\hline $\mathrm{AD} 1$ & $\mathrm{M}$ & 71 & 20 & 1.97 & 1340 & Pneumonia & Alzheimer's disease \\
\hline AD2 & $\mathrm{M}$ & 70 & $<24$ & 205 & 1150 & Pneumonia & Alzheimer's disease \\
\hline \multicolumn{8}{|c|}{ Normal (N 1-5) Controls } \\
\hline N 1 & $\mathrm{~F}$ & 80 & 9 & 2.27 & 1300 & $\begin{array}{l}\text { Peritoneal } \\
\text { carcinomatosis }\end{array}$ & Normal \\
\hline N 2 & $\mathrm{~F}$ & 82 & 3.5 & 0.79 & 1140 & Renal failure & Normal \\
\hline N 3 & $\mathrm{~F}$ & 71 & 24 & 2 & 1250 & $\begin{array}{r}\text { Hepatic surgery } \\
\text { complications }\end{array}$ & Normal \\
\hline $\mathrm{N} 4$ & $\mathrm{M}$ & 61 & 20 & 7 & 1420 & $\begin{array}{r}\text { Myocardial } \\
\text { Infarction }\end{array}$ & Normal \\
\hline N 5 & $\mathrm{M}$ & 53 & 24 & 2.17 & 1620 & Heart failure & Normal \\
\hline
\end{tabular}

${ }^{\text {a } P M I, ~ p o s t-m o r t e m ~ i n t e r v a l . ~}$

${ }^{\mathrm{b}}$ TIF, time in fix.

He died following an episode of acute severe congestive heart failure. At autopsy, his brain weighed $1130 \mathrm{~g}$. The left hemisphere was used for neuropathological diagnosis. There were numerous balloon cells with Pick morphology, positive for tau and ubiquitin, and numerous glial cytoplasmic inclusions. No Lewy bodies or Alzheimer pathology was identified. The neuropathological features were consistent with PiD. The right hemisphere was available for examination of the NBM, including the NSP, in the present study.

\section{Alzheimer's Disease (AD1)}

A 71-year-old right-handed male presented with progressive difficulty in word-finding for two and a half years. He did not have problems with oral or reading comprehension. He had no other clinical deficits or changes in his day-to-day function. His brain CT scan showed moderate diffuse cerebral atrophy. He was diagnosed with non-fluent agrammatic PPA. He subsequently developed dementia and behavioral disturbance and died due to pneumonia, 8 years after initial presentation. At autopsy, his brain weighed $1340 \mathrm{~g}$. The right hemisphere was used for neuropathological diagnosis. There was moderate to severe neuritic plaque density demonstrated with amyloid immunohistochemistry. There was also severe NFT deposition corresponding to a Braak stage of IV/VI AD. The left hemisphere was available for examination of the NBM, including the NSP, in the present study.

\section{Alzheimer's Disease (AD2)}

A 70-year-old male presented with progressive difficulty initiating speech. His speech was difficult to understand. A clinical diagnosis of PPA was made. He subsequently developed impaired comprehension and agitation. The patient died of aspiration pneumonia 8 years later. At autopsy, his brain weighed $1150 \mathrm{~g}$. There was moderate generalized atrophy. The left hemisphere was used for neuropathological diagnosis. There were up to 38 plaques per medium power field and NFTs with Braak stage VI/VI indicating a neuropathological diagnosis of AD. The left hemisphere was available for examination of the NBM, including the NSP, in the present study.

\section{Control Cases}

Five brains from individuals who did not have clinical or neuropathological diagnoses of $\mathrm{AD}$ or any other neurological disorder were chosen for comparison. These control cases were selected to approximately match cases based on age (Table 1), and the left hemispheres were examined.

\section{Tissue Preparation}

For each brain, the hemisphere used in the present study was cut into 1-2 cm coronal slabs and immersion fixed in $10 \%$ buffered formalin $(\mathrm{pH} 7.4)$ at $4{ }^{\circ} \mathrm{C}$ for $48-120 \mathrm{~h}$. Following fixation, all tissues were cryoprotected in a graded series of sucrose $(10,20,30$, and $40 \%)$ in $0.1 \mathrm{M}$ phosphate buffer (PB; $\mathrm{pH}$ 7.4), for approximately $48 \mathrm{~h}$ in each concentration, and stored in $40 \%$ sucrose with $0.6 \%$ sodium azide in PB at $4{ }^{\circ} \mathrm{C}$ until used.

Blocks of tissue obtained at the level of the NBM, including the NSP, were sectioned on a Leica SM2000R microtome (Leica Microsystems Inc., Nussloch, Germany) with Physitemp freezing stage and BFS-30TC controller (Physitemp Instruments, Inc., Clifton, NJ) into $50 \mu \mathrm{m}$ thick serial sections (PSP case was cut at $40 \mu \mathrm{m})$, and frozen at $-20{ }^{\circ} \mathrm{C}$ in $40 \%$ sucrose in PB until needed for immunohistochemical studies. For two PPA cases (VAD and PiD), sections were only available from the right hemisphere, and the left hemisphere had been used for neuropathological analysis at autopsy.

Starting at the level of the decussation of the anterior commissure and ending at the level of the mammillary bodies (Figure 1), eight sections (approximately $1 \mathrm{~mm}$ apart), were selected for ChAT immunohistochemical staining. Adjacent sections were stained for Nissl substance. 


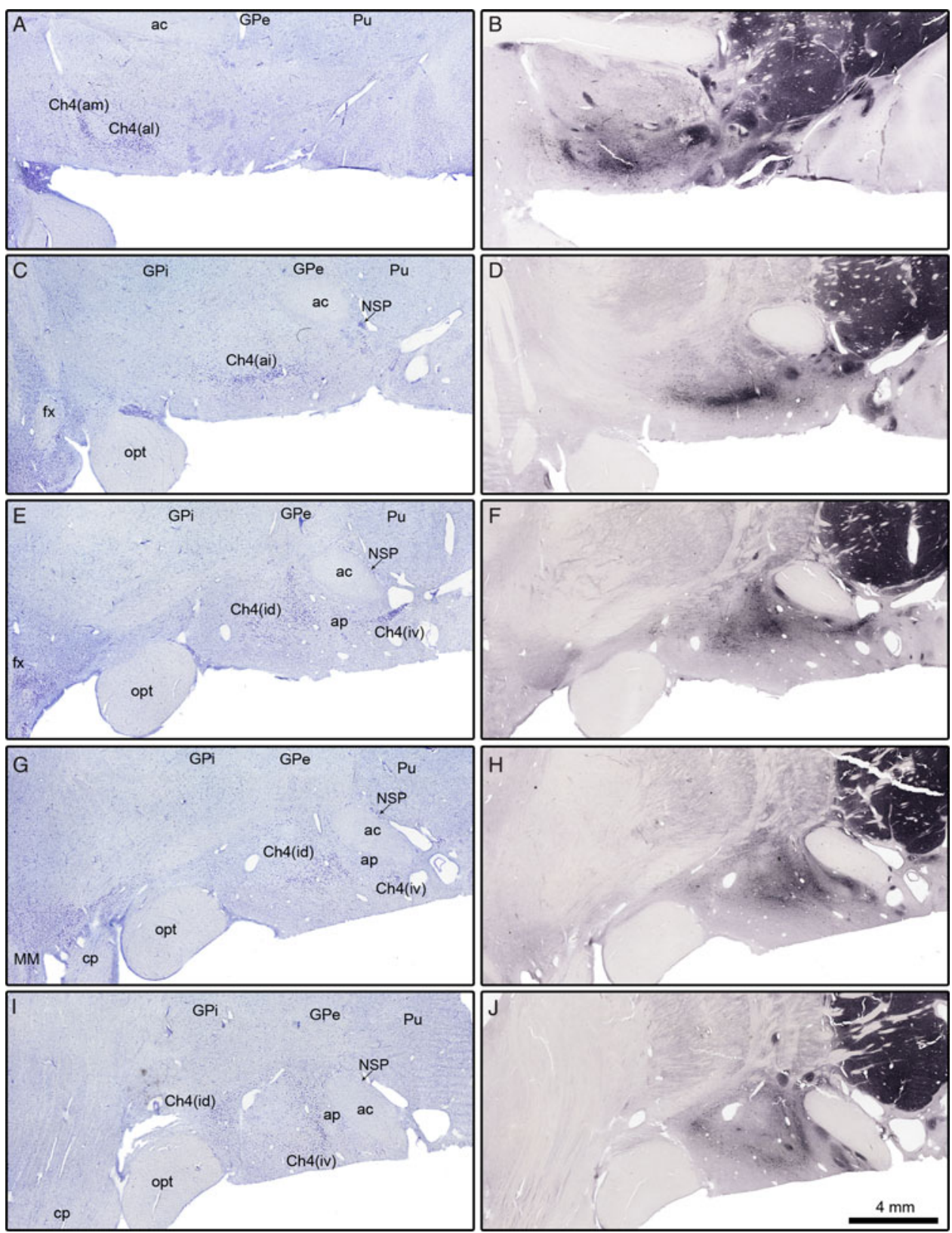

Figure1: The rostral (top) and more caudal (bottom) divisions of the human nucleus basalis of Meynert and nucleus subputaminalis (NSP), from case N5 stained for Nissl substance (left) and choline acetyltransferase (right). Note, both nuclei are visualized rostrally at the level of the decussation of the anterior commissure $(A$ and B). The NSP ends caudally at the mammillary level. Scale bar $=1 \mathrm{~mm}$. Abbreviations: ac, anterior commissure; ap, ansa peduncularis; Ch4(am), anteromedial-Ch4; Ch4(al), anterolateral-Ch4; Ch4(id), interomediodorsal-Ch4; Ch4(iv), interomedioventral-Ch4; Ch4ai, anterointermediate-Ch4; cp, cerebral peduncle; fx, fornix; GPe, globus pallidus external; GPi, globus pallidus internal; MM, mammillary body; opt, optic tract; Pu, putamen.

\section{Thionin Staining}

To examine cytoarchitecture, sections were stained for Nissl substance using thionin dye. Mounted sections were dehydrated in a series of ethanol washes (70-100\%), cleared in xylene, and rehydrated in a series of ethanol washes (100-70\%) followed by distilled water $\left(\mathrm{dH}_{2} \mathrm{O}\right)$. Sections were immersed in thionin dye for $3 \mathrm{~s}$ then rinsed in $\mathrm{dH}_{2} \mathrm{O}$, followed by a series of ethanol washes (70-100\%), then xylene and then coverslipped with an aqueous mounting medium.

\section{Immunohistochemical Staining}

Standard immunohistochemical staining procedures were conducted to detect ChAT. This was performed using previously employed and characterized primary antibody for ChAT (Millipore, AB144P, RRID\# AB_2079751).$^{28}$ All sections were rinsed for $30 \mathrm{~min}$ in $\mathrm{PB}$ then rinsed in diluted $\mathrm{PB}(0.05 \mathrm{M})$ followed by $\mathrm{dH}_{2} \mathrm{O}$ for $5 \mathrm{~min}$. Sections were then incubated in $0.3 \% \mathrm{H}_{2} \mathrm{O}_{2}$ in $\mathrm{PB}$ for $30 \mathrm{~min}$ to quench endogenous peroxidase activity and rinsed again for $30 \mathrm{~min}$ in $\mathrm{PB}$. Sections were incubated in $\mathrm{PB}$ 

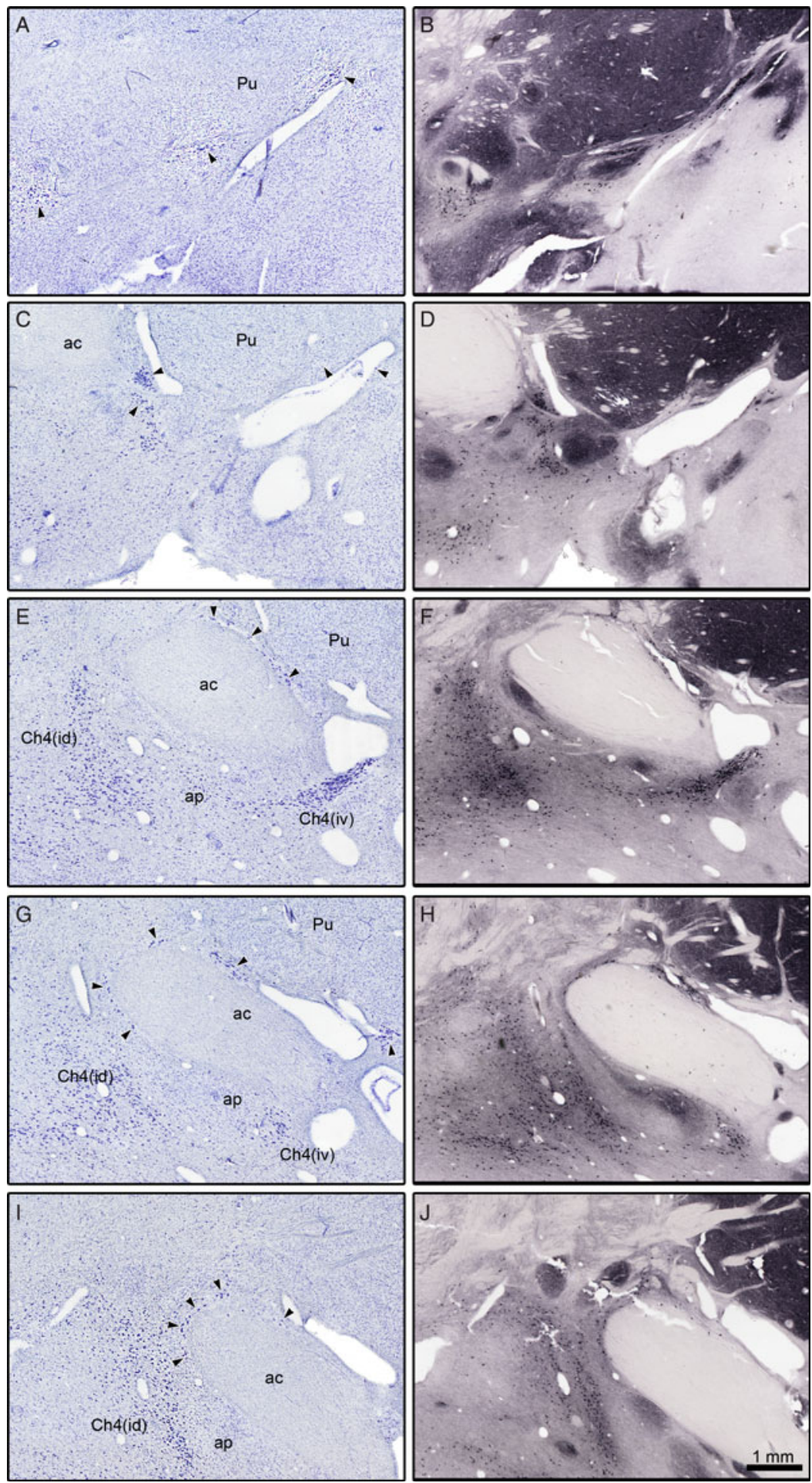

Figure 2: The rostral (top) and more caudal (bottom) human nucleus subputaminalis (NSP), from case N5 stained for Nissl substance (left) and choline acetyltransferase (right). Arrowheads denote NSP neurons. Note, in more rostral sections, the NSP is localized ventral to the putamen, in one or several clusters. At the intermediate level, the NSP is predominantly found lateral to the posterior limb of the anterior commissure and medial to the putamen. More caudally, as the anterior commissure recedes, NSP neurons are located dorsal to the posterior limb of the anterior commissure, medioventral to the putamen. Scale bar $=1 \mathrm{~mm}$. Abbreviations: ac, anterior commissure; ap, ansa peduncularis; Ch4(id), interomediodorsal-Ch4; Ch4(iv), interomedioventral-Ch4; Pu, putamen. 
Table 2: Total number and average number of cholinergic neurons in the nucleus basalis of Meynert (NBM) and nucleus subputaminalis (NSP) in progressive aphasia cases (PPA) and normal control (N) and percent reduction in these neurons in PPA cases compared to normal controls

\begin{tabular}{|c|c|c|c|c|c|c|c|c|c|c|c|c|}
\hline \multirow{3}{*}{$\begin{array}{l}\text { PPA/ } \\
\text { Normal }\end{array}$} & \multirow{3}{*}{$\begin{array}{c}\text { Number of } \\
\text { Sections } \\
\text { analyzed }\end{array}$} & \multicolumn{5}{|c|}{ NBM } & \multirow{3}{*}{$\begin{array}{c}\text { Number of } \\
\text { Sections } \\
\text { analyzed }\end{array}$} & \multicolumn{5}{|c|}{ NSP } \\
\hline & & \multicolumn{2}{|c|}{ Total Neuronal Counts } & \multicolumn{2}{|c|}{$\begin{array}{l}\text { Average Neuronal } \\
\text { Count/Section }\end{array}$} & \multirow[t]{2}{*}{$\begin{array}{c}\% \\
\text { Reduction } \\
\text { for PPA }\end{array}$} & & \multicolumn{2}{|c|}{ Total Neuronal Counts } & \multicolumn{2}{|c|}{$\begin{array}{c}\text { Average Neuronal } \\
\text { Count/Section }\end{array}$} & \multirow[t]{2}{*}{$\begin{array}{c}\% \\
\text { Reduction } \\
\text { for PPA }\end{array}$} \\
\hline & & PPA & $\mathbf{N}$ & PPA & $\mathbf{N}$ & & & PPA & $\mathbf{N}$ & PPA & $\mathbf{N}$ & \\
\hline $\mathrm{VaD} / \mathrm{N} 1$ & 6 & 4992 & 6062 & 832 & 1010 & 17.7 & 6 & 319 & 927 & 53 & 155 & 65.6 \\
\hline $\mathrm{PSP} / \mathrm{N} 2$ & 5 & 4114 & 5328 & 823 & 1066 & 22.8 & 5 & 243 & 478 & 49 & 96 & 49.3 \\
\hline $\mathrm{PiD} / \mathrm{N} 3$ & 6 & 3353 & 6903 & 559 & 1151 & 51.4 & 6 & 343 & 647 & 57 & 108 & 47.0 \\
\hline $\mathrm{AD} 1 / \mathrm{N} 4$ & 8 & 2518 & 10365 & 315 & 1296 & 75.7 & 6 & 172 & 582 & 29 & 97 & 70.4 \\
\hline $\mathrm{AD} 2 / \mathrm{N} 5$ & 8 & 2254 & 7690 & 282 & 961 & 70.7 & 8 & 128 & 1429 & 16 & 179 & 91.0 \\
\hline $\begin{array}{l}\text { Group } \\
\text { Mean } \\
\text { (SD) }\end{array}$ & - & - & - & $562(265)$ & $1097(132)$ & 47.7 & & - & - & 41 (18) & $127(38)$ & 64.7 \\
\hline
\end{tabular}

containing $0.1 \%$ Triton $\mathrm{X}-100$, normal rabbit serum (1:100), and goat anti-ChAT primary antibody for approximately $16 \mathrm{~h}$ at room temperature. Following another rinse in $\mathrm{PB}$ for $30 \mathrm{~min}$, sections were incubated in $\mathrm{PB}$ with $0.1 \%$ Triton $\mathrm{X}-100$, biotinylated rabbit anti-goat secondary antibody (1:500; Vector Labs), and normal goat serum $(1: 1000)$ for $1 \mathrm{~h}$ at room temperature. After another rinse in $\mathrm{PB}$, sections were placed in $\mathrm{PB}$ with $0.1 \%$ Triton $\mathrm{X}-100$ and Vectastain ${ }^{\circledR}$ Elite ABC kit (Vector) according to the manufacturer's instructions for $1 \mathrm{~h}$ at room temperature. After rinsing the sections in PB, they were devel-

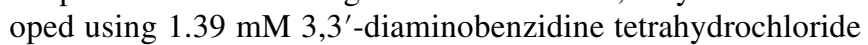
(DAB) in PB. After $5 \mathrm{~min}, 50 \mu \mathrm{L} 0.3 \% \mathrm{H}_{2} \mathrm{O}_{2}$ in $\mathrm{dH}_{2} \mathrm{O}$ was added per $\mathrm{mL}$ of $\mathrm{DAB}$ solution. The reaction was stopped by rinsing the sections in $0.01 \mathrm{M}$ acetate buffer ( $\mathrm{pH} 3.3$ ) for $30 \mathrm{~min}$. Control experiments were performed by omitting the primary or secondary antibody.

\section{Data Analysis}

Sections were analyzed and photographed using a Zeiss Axioplan 2 motorized brightfield microscope with a Zeiss Axiocam HRc digital camera with AxioVision 4.6 software (Carl Zeiss Canada Ltd, Toronto, Ontario, Canada) in the Cellular and Molecular Digital Imaging Facility, Dalhousie University (Halifax, Nova Scotia, Canada). All photographs were assembled using Adobe Photoshop CS5. Images were color balanced, contrast enhanced, and the brightness adjusted to match the background from different images.

ChAT-positive neurons were counted using brightfield microscopy with the Neurolucida software. At low magnification, the slide was outlined with a pen tool, and each subcortical structure was delineated. The tracings of the structures provided margins for labeling the neurons that were ventral to the putamen and medial to the anterior commissure. Then, at $100 \times$ magnification, all cells were visualized and those that were larger than $20 \mu \mathrm{m}$ and in the correct location were labeled with a red marker and regarded as part of the NBM and NSP (Figure 2). The total number of neurons was counted per case from the anterior to posterior extent of the nuclei. For consistency, the same method was used for counting neurons in all the cases.
The PPA cases were compared with control cases that were most closely matched by age and sections were obtained at the same rostrocaudal level for comparison. Due to technical issues with tissue staining, or missing sections during tissue extraction, some sections were not available in a few cases for analysis. Only sections at the same rostrocaudal level between PPA cases and controls were analyzed, therefore, the number of sections analyzed varied from 5 to 8 per PPA-control pair (Table 2). The total number of ChAT-positive cells in the NSP and NBM were compared between PPA and matched control cases and this was expressed as a percent reduction of cells in PPA versus normal controls. In addition, pooled analyses with 2-tailed unpaired independent samples $t$-tests were used to compare the PPA and normal control groups using the mean number of neurons observed in the NSP and NBM for each case and their matched control.

\section{ReSUlts}

\section{Localization of the Nucleus Subputaminalis}

All cholinergic cells ventral to the basal ganglia and anterior commissure and medial to the putamen were labeled and counted as the NBM (Figure 1). Differentiation of cells of the NSP from cells of the rest of the NBM was based on location of the neurons, with NSP neurons seen in aggregated clusters near the ventral border of the putamen (Figure 2), according to criteria described previously. ${ }^{16}$ Nissl staining allowed for the visualization of large, hyperchromatic neurons of various morphologies, including multipolar, spindle-shaped, and fusiform, belonging to the NSP. Rostrally, at the level of the decussation of the anterior commissure, NSP neurons could be seen at the ventrolateral border of the putamen (Figure 2). At the intermediate level, the NSP was lateral to the posterior limb of the anterior commissure and was medial as well as ventral to the putamen (Figure 2). Caudally, the NSP was ventral to the putamen and dorsolateral to the posterior limb of the anterior commissure, which had regressed (Figure 2). The cell population was delineated from the rostral-caudal extent using the decussation of the anterior commissure as the rostral landmark, 

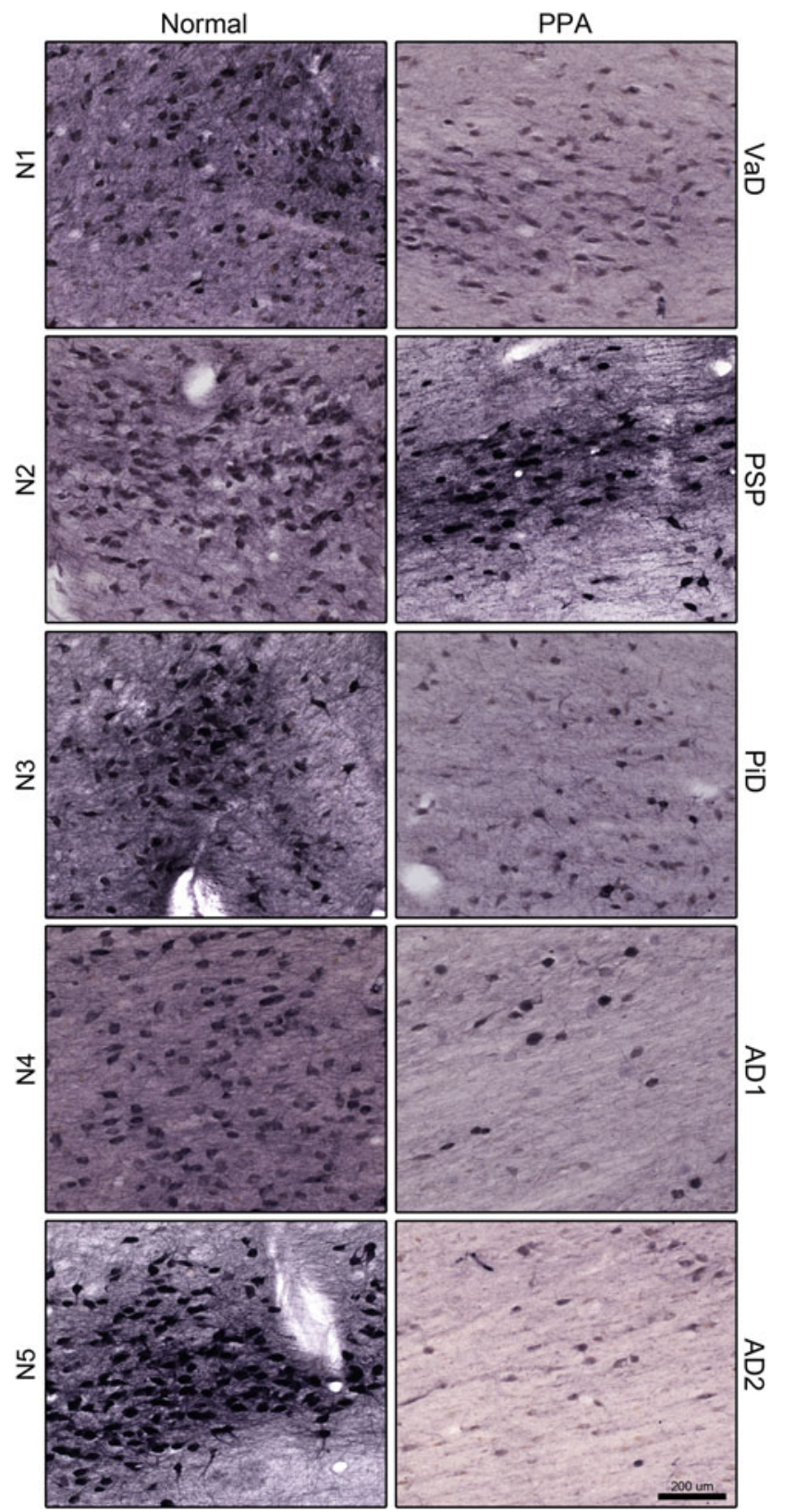

Figure 3: Choline acetyltransferase (ChAT)-positive neurons in the nucleus basalis of Meynert (NBM) Ch4 cell group, in normal (left) and primary progressive aphasia (PPA; right). Note, the marked decrease of ChAT-positive neurons in PPA. Scale bar $=200 \mu \mathrm{m}$. Abbreviations: AD, Alzheimer's disease; PiD, Pick's disease; PSP, progressive supranuclear palsy; VaD, vascular dementia.

and mammillary bodies as the caudal limit (Figure 1). All Ch4 sectors apart from NSP were considered as NBM for the purposes of our analyses.

\section{Cholinergic Neuronal Loss in the Nucleus Basalis of Meynert and Nucleus Subputaminalis}

In comparing the total number of cholinergic neurons in the NBM of the VaD case to the NBM in the control case, there was

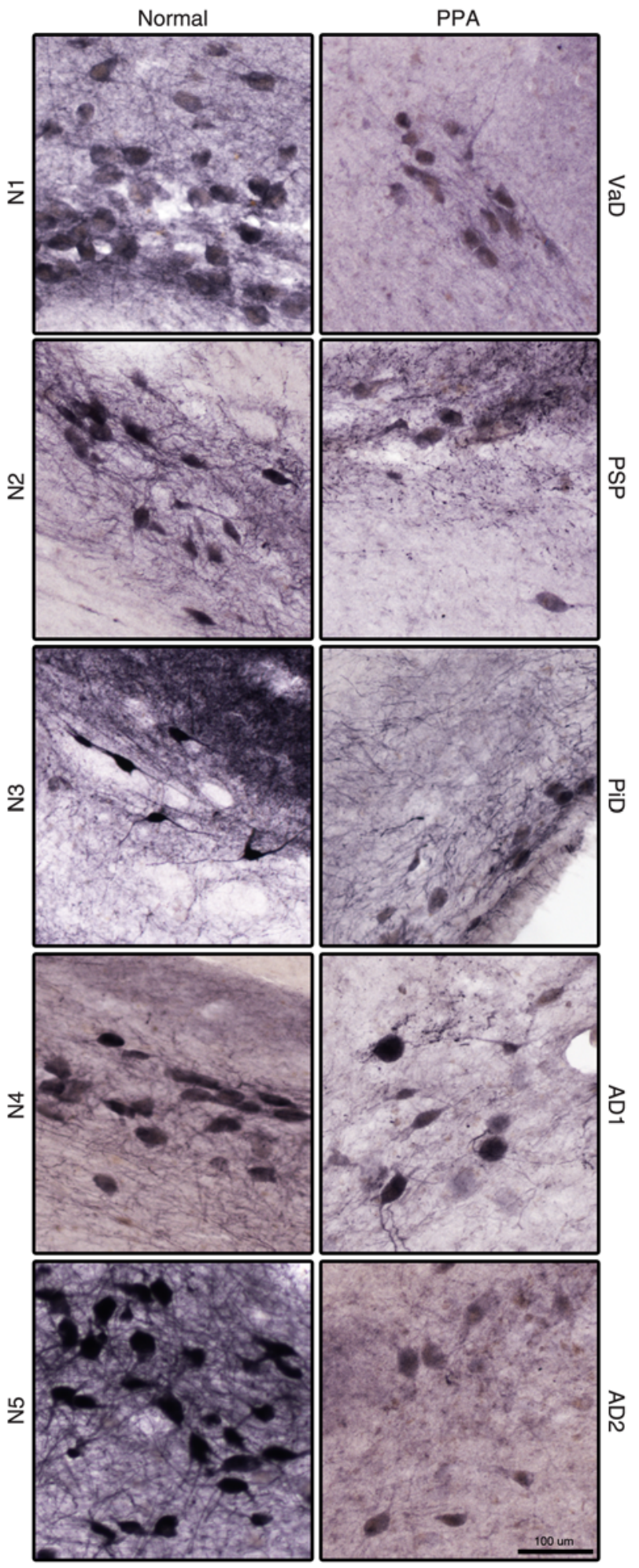

Figure 4: Choline acetyltransferase (ChAT)-positive neurons in the nucleus subputaminalis (NSP), in normal (left) and primary progressive aphasia (PPA; right). Note, the marked decrease of ChATneurons in the NSP in PPA. Scale bar $=100 \mu \mathrm{m}$. Abbreviations: AD, Alzheimer's disease; PiD, Pick's disease; PSP, progressive supranuclear palsy; VaD, vascular dementia. 
$17.7 \%$ reduction. Comparison of the PSP case with its matched control showed $22.8 \%$ reduction. In the $\mathrm{PiD}$ case, a similar comparison showed $51.4 \%$ reduction. The two AD cases showed $75.7 \%$ and $70.7 \%$ reduction, respectively. The total number of neurons and their respective percent reductions are shown in Table 2. Loss of cholinergic neurons in the NBM in all cases presenting as PPA was evident throughout the rostrocaudal extent of this NBM region. On average, there was a $47.7 \%$ reduction of cholinergic neurons in the NBM for PPA cases. A paired samples $t$-test showed a significant difference between normal and PPA groups in the NBM region, with assumed equal variance based on Levene's test, $t(8)=4.04, p=0.0037$. Further, Cohen's effect size value $(d=2.62)$ suggests a large significance. ${ }^{29}$

Cholinergic neurons in the NSP were analyzed using the same approach described above for the NBM. Thus, there was $65.6 \%$ reduction in $\mathrm{VaD}, 49.3 \%$ reduction in PSP, $47.0 \%$ reduction in $\mathrm{PiD}, 70.4 \%$ reduction in $\mathrm{AD} 1$, and $91.0 \%$ reduction in AD2 cases relative to their matched controls. The total number of neurons and their respective percent reductions are shown in Table 2. Loss of cholinergic neurons in the NBM (Figure 3 ) in all cases presenting as PPA was evident throughout the rostrocaudal extent of this NSP (Figure 4). On average, there was a $64.7 \%$ reduction of cholinergic neurons in the NSP for PPA cases. A paired samples $t$-test showed a significant difference between normal and PPA groups in the NSP region, with unequal variances assumed based on Levene's test, $t(6)$ $=4.62, p=0.0042$. Further, a Cohen's effect size value $(d=2.92)$ suggests a larger significance.

The PPA cases that displayed the greatest loss of cholinergic neurons in both the NBM and the NSP relative to their matched controls were those with AD pathology. In relation to their matched controls, three of the five PPA cases demonstrated a greater reduction in NSP cholinergic neurons as compared to NBM cholinergic neuronal loss. Of these, the PPA case with vascular pathology $(\mathrm{VaD})$ displayed the largest discrepancy between NBM cholinergic neuron reduction and NSP cholinergic neuron reduction $(17.7 \%$ vs. $65.6 \%$, respectively). The smallest discrepancy in the NBM versus NSP cholinergic neuron reduction was seen for the PiD case $(51.4 \%$ versus $47.0 \%)$.

\section{Discussion}

PPA is characterized by a progressive loss in language function, with relative sparing of other cognitive domains at initial stages of the condition. ${ }^{4,5,6}$ This clinical presentation is recognized as a consequence of a variety of neuropathological disease states, including $\mathrm{AD}, \mathrm{PiD}$, PSP, and CBD. ${ }^{13}$

Currently, the relationships between clinical PPA presentations and the pathology of specific PPA subtypes remain uncertain. In addition, despite consensus criteria for clinical subtyping, ${ }^{6}$ there are many patients who do not easily fit into these classifications. ${ }^{7,30,31}$ However, previous studies suggest that basal forebrain volumes are reduced in at least some of the PPA patients regardless of subtype and that neocortical acetylcholinesterase activity as measured using positron emission tomography (PET) differs between PPA and controls. ${ }^{14}$ This provides support for the involvement of basal forebrain subnuclei and the cholinergic system in PPA. We examined a nucleus within the basal forebrain postulated to be involved in language function, the NSP. The NSP is a cluster of neurons located ventral to the putamen that most likely corresponds to the $\mathrm{Ch} 4 \mathrm{p}$ region of NBM. ${ }^{16,17,23}$
The purpose of the present study was to determine whether the individuals who presented with a clinical diagnosis of PPA and developed different clinical and neuropathological profiles over time show a deficit in cholinergic neurons in the NSP. The results of our study provide evidence for the involvement of the NSP in every PPA case.

The case that showed microvascular pathology (VaD) but presented with PPA showed a relatively limited reduction of cholinergic neurons in the NBM (17.7\%), as compared to control, but showed a substantial reduction in cholinergic neurons in the NSP (65.6\%). This was despite the fact that in this case, the right hemisphere was examined. As there was no regionalization of the vascular changes seen, we expect that these vascular changes had a limited contribution to her initial presentation of primary progressive aphasia and posit that this component of her presentation was on the basis of neurodegenerative changes affecting the NSP.

One of our cases had a 4-year history of aphasia and pathology consistent with PSP. Aphasia can be a presenting symptom in PSP, leading to the initial diagnosis of PPA in some of these patients. ${ }^{32-36}$ Our PSP case showed a loss of cholinergic neurons in both the NBM and the NSP, but again showed greater cholinergic neuronal loss in the NSP $(22.8 \%$ reduction compared to $49.3 \%$; Table 2) relative to their matched control. In PSP, the loss of cholinergic neurons is most commonly found in the brainstem $^{37,38}$ although cholinergic neuronal loss has also been documented in the NBM. ${ }^{38,39}$ The fact that, in addition to a general loss of cholinergic neurons in the NBM, the NSP was more profoundly affected in our PSP case who presented with PPA further supports involvement of the NSP in language function.

The PiD case had a 6-year history of language impairment and an initial diagnosis of PPA but the pathological findings were consistent with $\mathrm{PiD}$ at autopsy. The PiD case described here showed a $51.4 \%$ reduction of cholinergic cells in the NBM compared to normal, and a $47.0 \%$ reduction of cholinergic neurons in the NSP compared to normal. This relatively similar reduction in cholinergic cells in the NBM and the NSP may have reflected the fact that only the right hemisphere was available for analysis. The left NSP is connected to the right NSP via projection fibers ${ }^{40}$ and a neurodegenerative process affecting the NSP in one hemisphere may well affect the homologous region in the other hemisphere, as occurs for other brain regions. ${ }^{40}$ Pick's pathology is seen in individuals with frontotemporal dementia (FTD) although our case did not meet clinical criteria for behavioral variant of FTD. ${ }^{41}$ Some patients with FTD present with language impairment at disease onset, ${ }^{6,41,42}$ but depletion of the cholinergic system is not characteristically associated with FTD. ${ }^{43-45}$ By contrast, loss of cholinergic neurons in the NBM has been documented in cases with Pick's pathology ${ }^{46-48}$ as was seen here. Regardless, in this case of PiD, who presented with a clinical diagnosis of PPA, the loss of cholinergic neurons in the NSP was substantial, as in our other PPA cases.

Two of our PPA cases ultimately had pathological findings consistent with AD. The finding of AD pathology in a subgroup of individuals with PPA has been well documented. ${ }^{6,49}$ As with the other PPA cases that we examined, loss of cholinergic neurons in the NSP was substantial for both AD cases, though one (AD1) showed a similar reduction in both the NBM and the NSP $(75.7 \%$ and $70.4 \%$, respectively), while the other (AD2) 
showed a slightly greater reduction of cholinergic neurons in the NSP (91.0\%) as compared to the NBM (70.7\%), relative to their matched control.

A limitation in the present study is a small sample size, due to the availability of few cases. In addition, our reports lacked sufficient clinical details to allow characterization of PPA into logopenic, agrammatic, or semantic subtypes. Furthermore, in two of our cases, only the right hemisphere was available for examination. Nevertheless, the findings of our preliminary study draw attention to a clear deficit in cholinergic neurons in the NSP of individuals presenting with PPA compared to controls. In particular, the extent of cholinergic cell loss seen in the NSP of the individuals with varying pathologies, but common presentations of progressive aphasia, may indicate how various distinct neuropathological processes can lead to similar clinical features, at least in terms of language deficits. We acknowledge that with such a small sample size, we cannot exclude the possibility that the loss of cholinergic neurons in the NSP of these cases may be due to mixed pathologies. Larger PPA samples that include distinct pathological groupings may further clarify the significance of the variance that we observed in the degree of cholinergic loss amongst our cases. Overall, we observed a significant loss of cholinergic neurons in the NSP and NBM, with relatively higher percentages of loss in the NSP when examining normal and PPA pairs. This suggests possible preferential involvement of the NSP in individuals presenting with progressive language disorders and provides grounds for further examination of the NSP as it relates to language dysfunction, and its involvement with cortical language networks.

\section{ACKNOWLeDGements}

All imaging and software for cell counts was made available through the CMDI/CORES facility. We would like to acknowledge Dr. Arnold Mitnitski and Drew DeBay for valuable discussions and Meghan Cash and Andrew Reid for their excellent technical support.

\section{FUNDING}

This work was supported in part by the Faculty of Medicine, Dalhousie University; Dalhousie Medical Research Foundation (Gunn Family Research Prize), the Dalhousie Medical Research Foundation Irene MacDonald Sobey Endowed Chair in Curative Approaches to Alzheimer's Disease, The Durland Breakthrough Fund, and the Dalhousie Faculty of Medicine Beth Rafuse Summer Studentship.

\section{Conflicts of InTEREST}

The authors confirm that this article content has no conflicts of interest.

\section{Ethical Approval}

Post-mortem human brain tissues were obtained from The Maritime Brain Tissue Bank (Halifax, Nova Scotia, Canada) following ethical approval from the Nova Scotia Health Authority Research Ethics Board.

\section{Statement of Authorship}

Authors contributed to the following aspects of the manuscript including project design ( $\mathrm{HH}$ and SD), performed experiments $(\mathrm{HH})$, data analysis (HH, JDF, and SD), manuscript preparation (HH, JDF, and SD), manuscript revision (HH, JDF, SD), and manuscript submission (HH, JDF, and SD).

\section{REFERENCES}

1. McKhann GM, Knopman DS, Chertkow H, et al. The diagnosis of dementia due to Alzheimer's disease: recommendations from the National Institute on Aging-Alzheimer's Association workgroups on diagnostic guidelines for Alzheimer's disease. Alzheimers Dement. 2011;7:263-9.

2. Kertesz A, McMonagle P, Blair M, Davidson W, Munoz DG. The evolution and pathology of frontotemporal dementia. Brain. 2005;128:1996-2005.

3. Santos-Santos MA, Mandelli ML, Binney RJ, et al. Features of patients with nonfluent/agrammatic primary progressive aphasia with underlying progressive supranuclear palsy pathology or corticobasal degeneration. JAMA Neurol. 2016;73:733-42.

4. Mesulam M. Slowly progressive aphasia without generalized dementia. Ann Neurol. 1982;11:592-8.

5. Mesulam M. Primary progressive aphasia. Ann Neurol. 2001; 49:425-32.

6. Gorno-Tempini ML, Hillis AE, Weintraub S, et al. Classification of primary progressive aphasia and its variants. Neurology. 2011; 76:1006-14.

7. Vadenberghe R. Classification of the primary progressive aphasias: principles and review of progress since 2011. Alzheimers Res Ther. 2016;8:1-9.

8. Chawluk JB, Mesulam M, Hurtig $\mathrm{H}$, et al. Slowly progressive aphasia without generalized dementia: studies with positron emission tomography. Ann Neurol. 1986;19:68-74.

9. Tyrrell PJ, Warrington EK, Frackowiak RSJ, Rossor MN. Heterogeneity in progressive aphasia due to focal cortical atrophy: a clinical and PET study. Brain. 1990;113:1321-36.

10. Abe $\mathrm{K}$, Ukita $\mathrm{H}$, Yanagihara $\mathrm{T}$. Imaging in primary progressive aphasia. Neuroradiology. 1997;39:556-9.

11. Westbury C, Bub D. Primary progressive aphasia: a review of 112 cases. Brain Lang. 1997;60:381-406.

12. Gorno-Tempini ML, Dronkers NF, Rankin KP, et al. Cognition and anatomy in three variants of primary progressive aphasia. Ann Neurol. 2004;55:335-46.

13. Mesulam M, Rogalski EJ, Wieneke C, et al. Primary progressive aphasia and the evolving neurology of the language network. Nat Rev Neurol. 2014;10:554-69.

14. Schaeverbeke J, Evenepoel C, Bruffaerts R, et al. Cholinergic depletion and basal forebrain volume in primary progressive aphasia. Neuroimage Clin. 2017;13:271-9.

15. Aarsland D, Larsen JP, Reinvang I, Aasland AM.Effects of cholinergic blockade on language in healthy young women: implications for the cholinergic hypothesis in dementia of the Alzheimer type. Brain. 1994;117:1377-84.

16. Simić G, Mrzljak L, Fucić A, Winblad B, Lovrić H, Kostović I. Nucleus subputaminalis (Ayala): the still disregarded magnocellular component of the basal forebrain may be human specific and connected with the cortical speech area. Neuroscience. 1998;89:73-89.

17. Mesulam M, Mufson EJ, Levey AI, Wainer BH. Cholinergic innervation of cortex by the basal forebrain: cytochemistry and cortical connections of the septal area, diagonal band nuclei, nucleus basalis (substantia innominata), and hypothalamus in the rhesus monkey. J Comp Neurol. 1983;214:170-97.

18. Mesulam M, Geula C. Nucleus basalis (Ch4) and cortical cholinergic innervation in the human brain: observations based on the distribution of acetylcholinesterase and choline acetyltransferase. J Comp Neurol. 1988;275:216-40.

19. Mesulam M, Van Hoesen GW. Acetylcholinesterase-rich projections from the basal forebrain of the rhesus monkey to neocortex. Brain Res. 1976;109:152-7. 
20. Mesulam M, Mufson EJ, Levey AI, Wainer BH. Atlas of cholinergic neurons in the forebrain and upper brainstem of the macaque based on monoclonal choline acetyltransferase immunohistochemistry and acetylcholinesterase histochemistry. Neuroscience. 1984;12:669-86.

21. Wolf D, Grothe M, Fischer FU, et al. Association of basal forebrain volumes and cognition in normal aging. Neuropsychologia. 2014;53:54-63.

22. Mufson EJ, Ginsberg SD, Ikonomovic MD, DeKosky ST. Human cholinergic basal forebrain: chemoanatomy and neurologic dysfunction. J Chem Neuroanat. 2003;26:233-42.

23. Ayala G. A hitherto undifferentiated nucleus in the forebrain (nucleus subputaminalis). Brain. 1915;37:433-48.

24. Raghanti MA, Simić G, Watson S, Stimpson CD, Hof PR, Sherwood CC.Comparative analysis of the nucleus basalis of Meynert among primates. Neuroscience. 2011;184:1-15.

25. Teipel SJ, Flatz W, Ackl N, et al. Brain atrophy in primary progressive aphasia involves the cholinergic basal forebrain and Ayala's nucleus. Psychiat Res. 2014;221:187-94.

26. Teipel S, Raiser T, Riedl L, et al. Atrophy and structural covariance of the cholinergic basal forebrain in primary progressive aphasia. Cortex. 2016;83:124-35.

27. Montine TJ, Phelps CH, Beach TG, et al. National institute on aging-Alzheimer's association guidelines for the neuropathologic assessment of Alzhiemer's disease: a practical approach. Acta Neuropathol. 2012;123:1-11.

28. Jarrett P, Easton A, Rockwood K, et al. Evidence for cholinergic dysfunction in autosomal dominant Kufs disease. Can J Neurol Sci. 2018: 45(2):150-7.

29. Cohen J. Statistical power analysis for the behavioral sciences. New York, NY: Routlege Academic; 1988.

30. Sajjadi SA, Patterson K, Arnold RJ, Watson PC, Nestor PJ. Primary progressive aphasia: a tale of two syndromes and the rest. Neurology. 2012;78:1670-7.

31. Wicklund MR, Duffy JR, Strand EA, Machulda MM, Whitwell JL, Josephs KA.Quantitative application of the primary progressive aphasia consensus criteria. Neurology. 2014;82:1119-26.

32. Deramecourt V, Lebert F, Debachy B, et al. Prediction of pathology in primary progressive language and speech disorders. Neurology. 2010;74:42-9.

33. Spaccavento S, Del Prete M, Craca A, Loverre A. A case of atypical progressive supranuclear palsy. Clin Interv Aging. 2014;9: 31-9.

34. Esmonde T, Giles E, Xuereb J, Hodges J. Progressive supranuclear palsy presenting with dynamic aphasia. J Neurol Neurosurg Psychiatry. 1996;60:403-10.
35. Boeve B, Dickson D, Duffy J, Bartleson J, Trenerry M, Petersen R. Progressive nonfluent aphasia and subsequent aphasic dementia associated with atypical progressive supranuclear palsy pathology. Eur Neurol. 2003; 49:72-8.

36. Josephs KA, Duffy JR. Apraxia of speech and nonfluent aphasia: a new clinical marker for corticobasal degeneration and progressive supranuclear palsy. Curr Opin Neurol. 2008;21:688-92.

37. Gilman S, Koeppe RA, Nan B, et al. Cerebral cortical and subcortical cholinergic deficits in parkinsonian syndromes. Neurology. 2010;74:1416-23.

38. Ruberg M, Javoy-Agid F, Hirsch E, et al. Dopaminergic and cholinergic lesions in progressive supranuclear palsy. Ann Neurol. 1985;18:523-9.

39. Tagliavini F, Pilleri G, Gemignani F, Lechi A. Neuronal loss in the basal nucleus of Meynert in progressive supranuclear palsy. Acta Neuropathol. 1983;61:157-60.

40. Petrides M,Pandya DN. Distinct parietal and temporal pathways to the homologues of Broca's area in the monkey. PLoS Biol. 2009;7(8):e1000170.

41. McKhann GM, Albert MS, Grossman M, Miller B, Dickson D, Trojanowski JQ. Clinical and pathological diagnosis of frontotemporal dementia: report of the Work Group on Frontotemporal Dementia and Pick's Disease. Arch Neurol. 2001;58:1803-9.

42. D’Anna L, Mesulam MM, Thiebaut de Schotten M, et al. Frontotemporal networks and behavioral symptoms in primary progressive aphasia. Neurology. 2016;86:1393-9.

43. Hirano S, Shinotoh H, Shimada H, et al. Cholinergic imaging in corticobasal syndrome, progressive supranuclear palsy and frontotemporaldementia. Brain. 2010;133:2058-68.

44. Lenz B, Sidiropoulos C, Bleich S, Kornhuber J. Frontotemporal dementia: neurotransmitter and clinical symptoms with focus on therapeutic targets. Fortschr Neurol Psychiatr. 2009;77:289-94.

45. Alonso-Navarro H, Jabbour-Wadih T, Ayuso-Peralta L, JiménezJiménez FJ. The neurochemistry and neuropharmacology of frontotemporal dementia. Rev Neurol. 2006;42:556-61.

46. Mizukami K, Kosaka K. Neuropathological study on the nucleus basalis of Meynert in Pick's disease. Acta Neuropathol. 1989; 78:52-6.

47. Sparks DL, Markesbery WR. Altered serotonergic and cholinergic synaptic markers in Pick's disease. Arch Neurol. 1991;48:796-9.

48. Uhl GR, Hilt DC, Hedrn JC, Whitehouse PJ, Price DL. Pick's disease (labor sclerosis), depletion of neurons in the nucleus basalis of Meynert. Neurology. 1983;33:1470-3.

49. Mesulam M, Wicklund A, Johnson N, et al. Alzheimer and frontotemporal pathology in subsets of primary progressive aphasia. Ann Neurol. 2008;6:709-19. 